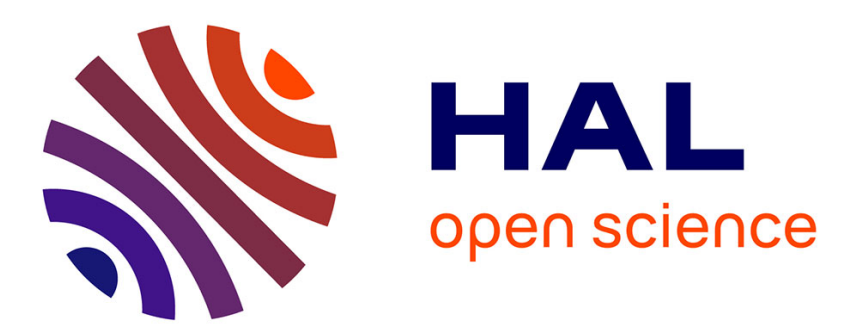

\title{
Modeling and control of flow with dynamical boundary actions
}

Andre Caldeira, Christophe Prieur, Daniel Coutinho, Valter Jùnior de Souza

Leite

\section{- To cite this version:}

Andre Caldeira, Christophe Prieur, Daniel Coutinho, Valter Jùnior de Souza Leite. Modeling and control of flow with dynamical boundary actions. MSC 2015 - IEEE Multi-Conference on Systems and Control, Sep 2015, Sydney, Australia. 10.1109/CCA.2015.7320835 . hal-01235437

\section{HAL Id: hal-01235437 \\ https://hal.science/hal-01235437}

Submitted on 30 Nov 2015

HAL is a multi-disciplinary open access archive for the deposit and dissemination of scientific research documents, whether they are published or not. The documents may come from teaching and research institutions in France or abroad, or from public or private research centers.
L'archive ouverte pluridisciplinaire HAL, est destinée au dépôt et à la diffusion de documents scientifiques de niveau recherche, publiés ou non, émanant des établissements d'enseignement et de recherche français ou étrangers, des laboratoires publics ou privés. 


\title{
Modeling and control of flow with dynamical boundary actions
}

\author{
André F. Caldeira, Christophe Prieur, Daniel Coutinho and Valter J. S. Leite
}

\begin{abstract}
In this paper a flow inside a pipe is modeled by a hyperbolic system (written in terms of a partial differential equation). One boundary condition is given by the coupling with a finite-dimensional dynamic model of heating column and a static modeling of ventilator. Then, the classical finite-dimensional technique is applied for the linearization of first order hyperbolic systems with dynamics associated to the boundary conditions. The discretization of the infinite-dimensional system is used and an augmented discrete linear system with dimension depending on the step size of discretization in space is obtained. The results are illustrated on simulations considering a Poiseuille flow experimental setup.
\end{abstract}

\section{INTRODUCTION}

This paper considers fluid transport which is a phenomenon often encountered in many industrial applications such as hydraulic networks [3], [14], gas flow in pipelines [1], [6], flow regulation in deep pits [15], among others. The fluid transport is modeled by conservation laws, which are first-order hyperbolic Partial Differential Equations (PDEs), commonly used to express the fundamental balance law that occur in many physical systems when small friction or dissipation effects are neglected [3]. It is well-known, that measurements and actuators in distributed parameter systems are not usually available. It is more usually for them to be located at the boundaries.

The stability problem of boundary control in time invariant hyperbolic systems has been attracted attention of academic community for a long time, see, e.g., the references [7], [8] and [12]. Moreover, a strict Lyapunov function approach is proposed in [13] for the boundary control with integral actions of hyperbolic systems of conservation law that can be diagonalized by means of Riemann invariants. The stability problem of linear and quasi-linear hyperbolic systems in the presence of dynamic behavior at the boundary conditions is addressed

André F. Caldeira is pursuing a doctoral degree on the Graduate Program on Systems and Control Engineering (PPGEAS) at the Universidade Federal de Santa Catarina (UFSC), PO BOX 476, 88040 - 900, Florianópolis, SC, Brazil and at GIPSA-lab, Grenoble, France. caldeiraaf@gmail. com

Christophe Prieur is with Department of Automatic Control, Grenoble Campus, Grenoble Image Parole Signal Automatique (GIPSA-lab), 11 rue de Mathématiques, B.P. 46, 38402 Saint Martin d'Heres, France. christophe.prieur@gipsa-lab.fr

Daniel Coutinho is with the DAS-CTC, UFSC, PO BOX 476, 88040 - 900, Florianópolis, SC, Brazil, and also with INESC P\&D Brazil. coutinho@das.ufsc.br

Valter J. S. Leite are with CEFET-MG/Campus Divinópolis, R. Álvares Azevedo, 400, 35503 - 822, Divinópolis, MG, Brazil. valter@iee.org in [5]. Reference [6] addresses the problem of boundary observer design for one-dimensional first order linear and quasi-linear strict hyperbolic systems with $n$ rightward convecting transport. The boundary control can have a faster reaction when compared to waves traveling time, because no time response limitation is taken into account at the boundary conditions. In certain applications such as [10], [2], the wave traveling can be considered much slower than the actuator time response. A static relationship between the control input and the boundary condition can be considered in many cases. However, there are applications where the dynamics associated with the boundary control cannot be neglected [4].

The major concern in this paper regards the modeling of a flow inside a pipe (fluid transport phenomenon) with boundary control strategy applied in a physical experimental setup. This problem is modeled by a nonlinear hyperbolic system with coupled equations and a boundary condition given by the coupling with a finitedimensional dynamic model of a heating column and a ventilator static model. The tracking problem of this complex dynamics is addressed in a simple manner by means of classical control tools such as linear approximations, finite difference schemes and integral action, leading to an augmented discrete-time linear system. Hence, a classical pole placement design methodology consisting of a state observer and a state feedback control law is applied to improve the closed-loop dynamics tracking performance. A numerical example considering the Poiseuille flow experimental setup is presented to illustrate the proposed strategy.

The rest of this paper can be summarized as follows. Section II introduces the description of fluid transport systems and the modeling of Poiseuille flow with dynamics at the boundary conditions. In Section III, it is presented an augmented discrete-time linear system obtained by means of linear approximation and spatial discretization with the inclusion of integral action for the steady-state tracking of constant reference signals. Section IV illustrates the proposed approach by means of a numeric example and Section V ends the paper.

\section{System Description And Modeling}

The fluid transport system is normally used for industrial applications. Such as ventilation system of mining industry [16] and hydraulic networks [14]. To investigate the phenomenon of fluid transport in a Poiseuille flow with dynamics at the boundary conditions, an experimental setup has been designed to test and validate 
control strategies. The Figure 1 shows the schematic of the proposed device.

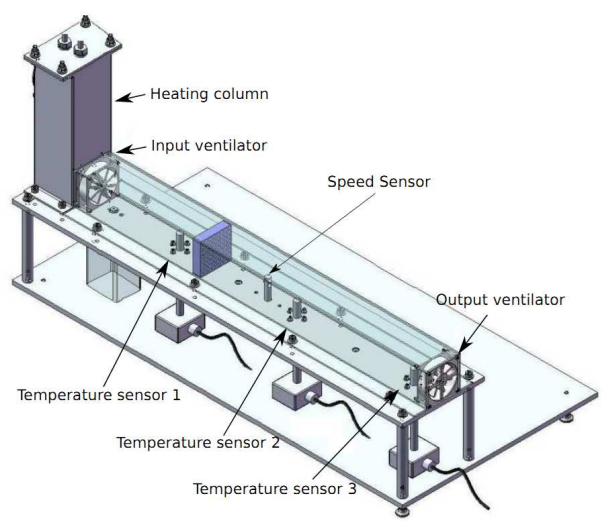

Fig. 1. Experimental setup (Poiseuille Flow)

This device is constituted by a heating column encasing a resistor, a tube, two ventilators, a gas speed meter and three distributed temperature sensors. The control objective is the outlet temperature tracking by driving the power dissipated on the heating resistor at different air flow speeds through the tube (input ventilator). Only the outlet temperature, the outlet flow speed, the outlet pressure and the heating column temperature will be considered as measurements for a closed-loop boundary control strategy. In this work, it is assumed that only the first ventilator (input ventilator) is the actuator and the output ventilator is set to be off.

The modeling of the experimental setup is done by considering three subsystems: the tube, the heating column and the ventilator. One-dimensional transport model is used to describe the gas density, speed and pressure variations in the tube. For the dynamic boundary conditions, it is considered a zero-dimensional model of control volume approach with heat exchanges coming from the heating resistor in the column. In addition, the Bernoulli's equation is used to relate the pressure, temperature and speed with the ventilator rotation for the static boundary condition, and the perfect gases law is used to convert density on temperature.

\section{A. Heating Column Model}

The control volume is based on reference [4]. Fig. 2 represents a control volume approach for the heating column.

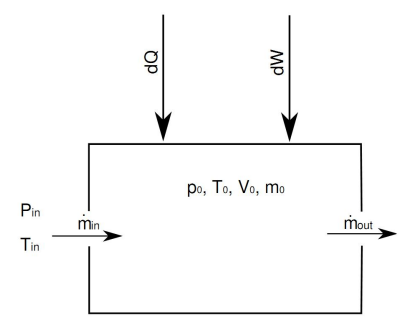

Fig. 2. Control volume approach
Consider the internal energy of a perfect gas:

$$
U_{0}=C_{v} m_{0} T_{0}
$$

where $U_{0}$ is the gas internal energy, $T_{0}$ is the gas temperature, $m_{0}$ is the mass inside the column and $C_{v}$ is the specific heat of the gas for constant volume. The time derivative of (1) is:

$$
\dot{U}_{0}=C_{v} m_{0} \dot{T}_{0}+C_{v} T_{0} \dot{m}_{0} .
$$

Using the first law of thermodynamics, the internal energy of the gas inside the column can be alternatively given by:

$$
\dot{U}_{0}=h_{\text {in }} \dot{m}_{\text {in }}+h_{\text {out }} \dot{m}_{\text {out }}+d Q+d W,
$$

where $h_{\text {in }}$ and $h_{\text {out }}$ are the specifics enthalpy getting in and out of the volume with a mass flow rate $\dot{m}_{i n}$ and $\dot{m}_{\text {out }}$, respectively. $d Q$ quantifies the heat exchanges and $d W$ is the work done by the gas. In the case of the heating column, there are two flows interacting with the volume, the input mass flow rate $\dot{m}_{i n}$ and the output mass flow rate $\dot{m}_{\text {out }}$. As the gas does not perform any work, then $d W=0$. In order to write (3) in terms of temperature, the specific enthalpy of a gas is used defined by $h=$ $C_{p} T$ with $C_{p}$ being the specific heat constant at constant pressure. Therefore, (3) can be redefined as:

$$
\dot{U}_{0}=C_{p} T_{i n} \dot{m}_{i n}-C_{p} T_{0} \dot{m}_{\text {out }}+d Q,
$$

where $T_{i n}$ is the heating column input temperature. To simplify the model, consider the following two hypothesis:

H1.1 The pressure dynamics in the heating column is much faster than the temperature dynamics, which allows a quasi-static behavior of the mass and pressure to be considered;

H1.2 $p_{0} \approx p_{i n}$, where $p_{i n}$ is the input pressure and $p_{0}$ is the pressure inside the column.

Thus, H1.1 and H1.2 allow (2) and (4) to be respectively rewritten as:

$$
\begin{gathered}
\dot{U}_{0}=C_{v} m_{0} \dot{T}_{0}, \\
\dot{U}_{0}=C_{p} \dot{m}_{i n}\left(T_{i n}-T_{0}\right)+d Q .
\end{gathered}
$$

For simplicity, the temperature dynamics can be expressed in terms of the gas density by introducing the following change of variable (perfect gases law):

$$
\rho_{0}=\frac{p_{\text {in }}}{R T_{0}},
$$

where $R$ is the ideal gas constant. Taking the time derivative of (7) into account, the following holds:

$$
\dot{T}_{0}=-\frac{R}{p_{\text {in }}} T_{0}^{2} \dot{\rho}_{0} .
$$

In addition, equalizing (5) and (6) and using (7)-(8) along with the perfect gases law to replace the mass inside the control volume $m_{0}$ in terms of the pressure and the specific gas constant $R$, it is obtained:

$$
\dot{\rho}_{0}=-\frac{R \gamma T_{i n} \dot{m}_{i n}}{p_{i n} V_{0}} \rho_{0}-\frac{R}{p_{i n} V_{0} C_{v}} \rho_{0} d Q+\frac{\gamma \dot{m}_{i n}}{V_{0}},
$$

where $\rho_{0}=\frac{m_{0}}{V_{0}}$ is the density inside the heating column, $V_{0}$ is the column volume and $\gamma=\frac{C_{p}}{C_{v}}$. 


\section{B. Flow model in the tube}

Consider the ideal gas flow through a constant section, where all the friction losses and heat transfers are neglected. Hence, the Euler equations can be used to model the system. The equations consist of conservation of mass, momentum and energy and they can be written in terms of the primitive variables density $\rho$, particle speed $v$ and pressure $p$. In this case, the flow is assumed to be one-dimensional leading to the following system of PDEs (which are three nonlinear coupled equations):

$$
\mathbf{W}_{t}+\mathbf{A} \mathbf{W}_{x}=\mathbf{0},
$$

where $\mathbf{W}=\left(\begin{array}{c}\rho \\ v \\ p\end{array}\right)$ and $\mathbf{A}=\left(\begin{array}{ccc}v & \rho & 0 \\ 0 & v & \frac{1}{\rho} \\ 0 & a^{2} \rho & v\end{array}\right)$, with $a=\sqrt{\frac{\gamma p}{\rho}}$ representing the sound speed in ideal gas and $\gamma$ the adiabatic constant. Further, $t \geq 0$ is the time variable and $x \in[0, L]$ is the space variable with $L=1.5$ being the tube length.

\section{Ventilator Model}

The ventilator is a power driven machine that moves a continuous volume of air by converting rotational mechanical energy to an increase in the total pressure of the moving air. From [11], there are three relationships among fan pressure, speed, power and flow rate, which are stated as ventilator laws. In this work, it is only considered one ventilator installed at the input side of the tube as the actuator, a flow rate proportional to the ventilator speed. Thus, the ventilator rotating speed is the control action.

Bernoulli's equation is used to relate the density, speed and pressure with the ventilator rotation. Using the ventilator law, the boundary condition is modeled, for $x=0$ and $t \geq 0$, by means of:

$$
p_{a}+\frac{1}{2} \rho(0, t) v(0, t)^{2}=K_{n} C(t)^{2}
$$

where the $p_{a}$ is the atmospheric pressure, $K_{n}$ is a constant coefficient, $C(t)$ is the ventilator rotating speed, and $\rho(0, t)$ and $v(0, t)$ represent gas density and velocity at the input side of tube, respectively.

\section{Outputs $T(L, \cdot), v(L, \cdot), p(L, \cdot)$ and $T(0, \cdot)$}

The outputs considered in this work are the particle temperature, speed and pressure at the outlet of the tube, respectively, $T(L, \cdot), v(L, \cdot)$ and $p(L, \cdot)$. The dynamic boundary condition is the temperature in the heating column $T(0, \cdot)$. The outputs $v(L, \cdot)$ and $p(L, \cdot)$ are obtained directly, but the outputs $T(L, \cdot)$ and $T(0, \cdot)$ are obtained by means of the nonlinear relationship (7). The pressure inside the tube is considered constant (equal to the atmospheric pressure, $\left.p_{a}\right)$. The pressure at the inlet $p_{\text {in }}$ and outlet $p_{\text {out }}$ of the tube are considered equal, since the pressure differential introduced by the ventilator is supposed to be small.

\section{Augmented Discrete-Time System}

This section is composed of three main steps: $(i)$ the linearization of the coupled nonlinear hyperbolic system (flow model in the tube), the nonlinear ordinary differential equation (ODE) that represents the heating column, the Bernoulli's equation for the ventilator model and the nonlinear relationship between density and temperature; (ii) discretization of the linear system resulting from the first step; and (iii) the representation of the system dynamics in terms of an augmented finite-dimensional discrete-time system, and the development of stability analysis and control design conditions.

\section{A. Linearization}

1) Flow model in the tube: system (10) admits a steady-state $\left(\rho^{*}, v^{*}, p^{*}\right)$ and the deviations of the states $(\rho, v, p)$ with respect to their steady-state values are defined as $\bar{\rho}=\rho-\rho^{*}, \bar{v}=v-v^{*}, \bar{p}=p-p^{*}$.

Thus, the linearization of system (10) at this equilibrium is given by

$$
\overline{\mathbf{W}}_{t}+\mathbf{A}^{*} \overline{\mathbf{W}}_{x}=\mathbf{0}
$$

with $\overline{\mathbf{W}}=\left(\begin{array}{c}\bar{\rho} \\ \bar{v} \\ \bar{p}\end{array}\right)$ and $\mathbf{A}^{*}=\left(\begin{array}{ccc}v^{*} & \rho^{*} & 0 \\ 0 & v^{*} & \frac{1}{\rho^{*}} \\ 0 & a^{* 2} \rho^{*} & v^{*}\end{array}\right)$.

2) Heating Column Model: whereas the pressure inside the tube is constant (and equal to the atmospheric pressure), the input mass flow rate will be expressed as

$$
\dot{m}_{i n}=v(0, t) \rho(0, t) A_{t},
$$

where $A_{t}$ is the tube cross section area.

Taking (13) into account, the boundary condition (9) can be expressed as:

$$
\begin{aligned}
\dot{\rho}(0, t)= & -\frac{R \gamma T_{i n} v(0, t) \rho(0, t) A_{t}}{p_{i n} V_{0}} \rho(0, t) \\
& -\frac{R}{p_{i n} V_{0} C_{v}} \rho(0, t) d Q+\frac{\gamma v(0, t) \rho(0, t) A_{t}}{V_{0}} .
\end{aligned}
$$

Defining

$$
\mathcal{A}=-\frac{R \gamma T_{i n} A_{t}}{p_{\text {in }} V_{0}}, \mathcal{B}=-\frac{R}{p_{i n} V_{0} C_{v}} \text { and } \mathcal{C}=\frac{\gamma A_{t}}{V_{0}},
$$

we obtain:

$$
\begin{aligned}
\dot{\rho}(0, t)=\mathcal{A} v(0, t) \rho(0, t)^{2}+\mathcal{B} d Q & \rho(0, t) \\
& +\mathcal{C} v(0, t) \rho(0, t) .
\end{aligned}
$$

Then, linearizing (15) and defining $d Q=U_{2}(t)$ yields:

$$
\begin{aligned}
\dot{\bar{\rho}}(0, t)=\mathcal{A}\left(\bar{v}(0, t) \rho^{* 2}\right. & \left.+2 v^{*} \rho^{*} \bar{\rho}(0, t)\right) \\
+\mathcal{B}\left(U_{2}^{*} \bar{\rho}(0, t)+\rho^{*} U_{2}(t)\right) & \\
& +\mathcal{C}\left(v^{*} \bar{\rho}(0, t)+\rho^{*} \bar{v}(0, t)\right),
\end{aligned}
$$

where $\bar{\rho}(0, t)=\rho(0, t)-\rho^{*}$ is a small deviation around the equilibrium point; $\rho^{*}$ and $v^{*}$ are the same equilibrium points used for flow model in the tube; and $U_{2}^{*}$ is a constant control action at the equilibrium point. 
Hence, reorganizing (16) leads to

$$
\begin{aligned}
\dot{\bar{\rho}}(0, t)=\left(2 v^{*} \rho^{*} \mathcal{A}+\mathcal{B} U_{2}^{*}+\mathcal{C} v^{*}\right) \bar{\rho}(0, t) \\
+\left(\mathcal{A} \rho^{* 2}+\mathcal{C} \rho^{*}\right) \bar{v}(0, t)+\mathcal{B} \rho^{*} U_{2}(t) .
\end{aligned}
$$

3) Ventilator Model: linearizing equation (11) assuming that $p_{a}$ is constant, the following boundary condition is derived:

$$
\bar{\rho}(0, t) v^{* 2}+2 \rho^{*} v^{*} \bar{v}(0, t)=4 K_{n} C^{*} \bar{C}(t),
$$

and then defining $\bar{C}(t)=U_{1}(t), C^{*}=U_{1}{ }^{*}$ and reorganizing (18) yields

$$
\bar{v}(0, t)=\left(-\frac{v^{*}}{2 \rho^{*}}\right) \bar{\rho}(0, t)+\left(\frac{4 K_{n} U_{1}^{*}}{2 \rho^{*} v^{*}}\right) U_{1}(t),
$$

with $U_{1}{ }^{*}$ being a constant control action at the equilibrium point.

4) Outputs $T(L, \cdot), v(L, \cdot), p(L, \cdot)$ and $T(0, \cdot)$ : the linear output $\bar{v}(L, \cdot)$ and $\bar{p}(L, \cdot)$ are obtained directly from the linear flow model in the tube. It is required to use the linearization of (7) to deal with $\bar{T}(L, \cdot)$ and $\bar{T}(0, \cdot)$, hence the following relationship is obtained

$$
\bar{T}(L, t)=-\frac{p_{\text {out }} \bar{\rho}(L, t)}{R \rho^{* 2}}, \quad \bar{T}(0, t)=-\frac{p_{\text {in }} \bar{\rho}(0, t)}{R \rho^{* 2}} .
$$

\section{B. Discretization}

1) Flow model in the tube: in order to spatially discretize the linear hyperbolic system (12), we shall make use of the forward and backward difference quotients for $\mathbf{W}_{t}$ and $\mathbf{W}_{x}$, respectively.

Let $\triangle t$ and $\triangle x$ be respectively the time and space steps satisfying a CFL condition [9]. Then, by routine manipulations over $j=0,1, \ldots, N$ and $i=1,2, \ldots, \alpha$ with $N$ being a given integer and $\alpha=\frac{L}{\triangle x}$, the PDE (12) is approximated by means of

$$
\begin{gathered}
\bar{\rho}_{d}(i, j+1)=b_{1} \bar{\rho}_{d}(i, j)+b_{2} \bar{\rho}_{d}(i-1, j) \\
-b_{3} \bar{v}_{d}(i, j)+b_{3} \bar{v}_{d}(i-1, j), \\
\bar{v}_{d}(i, j+1)=b_{1} \bar{v}_{d}(i, j)+b_{2} \bar{v}_{d}(i-1, j) \\
-b_{4} \bar{p}_{d}(i, j)+b_{4} \bar{p}_{d}(i-1, j),
\end{gathered}
$$

$$
\begin{aligned}
\bar{p}_{d}(i, j+1)=b_{1} \bar{p}_{d}(i, j)+b_{2} & \bar{p}_{d}(i-1, j) \\
& -b_{5} \bar{v}_{d}(i, j)+b_{5} \bar{v}_{d}(i-1, j),
\end{aligned}
$$

where $b_{1}=\left(1-\frac{\triangle t}{\triangle x} v^{*}\right), b_{2}=\left(\frac{\triangle t}{\triangle x} v^{*}\right), b_{3}=$ $\left(\frac{\triangle t}{\triangle x} \rho^{*}\right), b_{4}=\left(\frac{\triangle t}{\triangle x \rho^{*}}\right)$ and $b_{5}=\left(\frac{\triangle t}{\triangle x} \gamma p^{*}\right)$.

2) Ventilator Model: the discretization of (19) is obtained by applying a standard discretization $j \triangle t$ leading to

$$
\bar{v}_{d}(0, j)=\left(-\frac{v^{*}}{2 \rho^{*}}\right) \bar{\rho}_{d}(0, j)+\left(\frac{4 K_{n} U_{1}^{*}}{2 \rho^{*} v^{*}}\right) U_{1}(j) .
$$

3) Heating Column Model: considering $j \Delta t$, the discretization of the heating column liner approximation in (17) is as follows

$$
\begin{gathered}
\bar{\rho}_{d}(0, j+1)=\left(1+\Delta t\left(2 v^{*} \rho^{*} \mathcal{A}+\mathcal{B} U_{2}^{*}+\mathcal{C} v^{*}\right)\right) \overline{\rho_{d}}(0, j) \\
+\triangle t\left(\mathcal{A} \rho^{* 2}+\mathcal{C} \rho^{*}\right) \bar{v}_{d}(0, j)+\triangle t\left(\mathcal{B} \rho^{*}\right) U_{2}(j)
\end{gathered}
$$

with $\Delta t$ being the period of sampling. Thus, in view of the above, we derive the following expression by replacing $\bar{v}_{d}(0, j)$ as $(24)$ and reorganizing $(25)$

$\overline{\rho_{d}}(0, j+1)=Q_{1} \overline{\rho_{d}}(0, j)+Q_{2} U_{1}(j)+Q_{3} U_{2}(j), \quad(26)$

where $Q_{1}=\left(\begin{array}{c}\left(1+\Delta t\left(2 v^{*} \rho^{*} \mathcal{A}+\mathcal{B} U_{2}^{*}+\mathcal{C} v^{*}\right)\right. \\ +\left(\left(\triangle t\left(\mathcal{A} \rho^{* 2}+\mathcal{C} \rho^{*}\right)\right)\left(-\frac{v^{*}}{2 \rho^{*}}\right)\right)\end{array}\right)$,

$Q_{2}=\left(\left(\triangle t\left(\mathcal{A} \rho^{* 2}+\mathcal{C} \rho^{*}\right)\right)\left(\frac{4 K_{n} U_{1}^{*}}{2 \rho^{*} v^{*}}\right)\right)$ and $Q_{3}=$ $\triangle t \mathcal{B} \rho^{*}$

4) Outputs $T(L, \cdot), v(L, \cdot), p(L, \cdot)$ and $T(0, \cdot)$ : the discrete linear approximate outputs $\bar{v}_{d}(L, \cdot)$ and $\bar{p}_{d}(L, \cdot)$ are obtained directly from the discrete linear approximate flow model in the tube. For $\bar{T}(L, t)$ and $\bar{T}(0, t)$ as described in (20) is applied the standard discretization step $j \triangle t$ leading to:

$$
\bar{T}_{d}(0, j)=-\frac{p_{\text {in }} \bar{\rho}_{d}(0, j)}{R \rho^{* 2}}, \bar{T}_{d}(L, j)=-\frac{p_{\text {out }} \bar{\rho}_{d}(L, j)}{R \rho^{* 2}} .
$$

\section{Discrete-Time Linear Approximation}

After the steps of linearization and discretization, an augmented discrete-time linear model can be obtained. To this end, let the augmented state vector be defined by:

$$
\begin{aligned}
\mathcal{X}(j)=\left[\begin{array}{llll}
\bar{\rho}_{d}(1, j) & \bar{\rho}_{d}(2, j) & \cdots & \bar{\rho}_{d}(\alpha, j) \\
\bar{v}_{d}(1, j) & \bar{v}_{d}(2, j) & \cdots & \bar{v}_{d}(\alpha, j) \\
\bar{p}_{d}(1, j) & \bar{p}_{d}(2, j) & \cdots & \bar{p}_{d}(\alpha, j)
\end{array}\right]^{T},
\end{aligned}
$$

and the boundary points as follows

$$
\mathcal{X}_{c}(j)=\left[\begin{array}{ll}
\bar{\rho}_{d}(0, j) & \bar{v}_{d}(0, j)
\end{array}\right]^{T} .
$$

The boundary condition $\bar{p}_{d}(0, j)$ is considered constant and it is not used for the boundary control problem.

Since the boundary control is not directly applied to the states components density $\bar{\rho}_{d}(0, j)$ and speed $\bar{v}_{d}(0, j)$, it is employed the ventilator speed $U_{1}(j)$ (installed in the initial part of the tube) and the power dissipated on the heating resistor $U_{2}(j)$ (installed in the heating column) as the boundary control actuation. Thus, the control input vector of the discrete-time approximation is defined as:

$$
U(j)=\left[\begin{array}{ll}
U_{1}(j) & U_{2}(j)
\end{array}\right]^{T} .
$$

Notice that we have a static boundary condition and a dynamic boundary condition. Hence, to obtain the boundary condition related to the density $\bar{\rho}_{d}(0, j)$, we use the dynamic model of the heating column. Therefore, the augmented state vector is redefined as follows

$$
\mathcal{Z}(j)=\left[\begin{array}{c}
\mathcal{X}(j) \\
\bar{\rho}_{d}(0, j)
\end{array}\right]
$$


Then, the discrete-time linear approximation will be as follows:

$$
\Omega:\left\{\begin{aligned}
\mathcal{Z}(j+1) & =A \mathcal{Z}(j)+B U(j), \\
y(j) & =C \mathcal{Z}(j)
\end{aligned}\right.
$$

where $j$ is the sampling time index; $\mathcal{Z}(j) \in \mathbb{R}^{3 \alpha+1}$ is the augmented state vector; $U(j) \in \mathbb{R}^{m}$ represents the control input vector; $y(j) \in \mathbb{R}^{p}$ represents the output signals; and $A, B$ and $C$ are constant matrices as defined in (33) at the top of next page.

To implement a state feedback control law, a standard Luenberger-like observer is designed. That is, the following control law is considered

$$
U(j)=K \hat{\mathcal{Z}}(j),
$$

where $\hat{\mathcal{Z}}(j)$ is the state of the following observer

$$
\hat{\Omega}:\{\hat{\mathcal{Z}}(j+1)=(A-L C) \hat{\mathcal{Z}}(j)+B U(j)+L y(j),
$$

with $K \in \mathbb{R}^{m \times 3 \alpha+1}$ and $L \in \mathbb{R}^{3 \alpha+1 \times p}$ to be determined such that $(A-B K)$ and $(A-L C)$ are Schur stable.

\section{A Numerical Example}

Let the following system parameters: adiabatic constant $\gamma=1.4$; molar mass of dry air $M=28.97 \mathrm{~g} / \mathrm{mol} . \mathrm{K}$; ideal gas constant $R=8.3143 \mathrm{~J} /(\mathrm{mol} . K)$; specific heat constant for constant pressure $C_{p}=1.005 \mathrm{KJ} / \mathrm{Kg} . \mathrm{K}$ for constant volume $C_{v}=0.718 \mathrm{KJ} / \mathrm{Kg} . \mathrm{K}$; initial pressure $p_{\text {in }}=1 \times 10^{5} \mathrm{~Pa}$; initial temperature $T_{\text {in }}=304 \mathrm{~K}$; column volume $V_{0}=4 \times 10^{-3} \mathrm{~m}^{3}$; tube cross section area $A_{t}=6.4 \times 10^{-3} \mathrm{~m}^{2}$; tube length $L=1.5 \mathrm{~m}$; and the constant coefficient of ventilator model $K_{n}=1 \times 10^{-4}$. In addition, define the discretization steps as $\triangle t=0.0075$ and $\triangle x=0.036$ which ensure the numerical stability condition of [9] (i.e., $b_{1}<|1|$ - with $b_{1}=0.5032$ ).

Firstly, some simulations are performed aiming to validate the proposed models, i.e., the hyperbolic nonlinear system (10) with nonlinear boundary conditions (9) and (11) referred as $H S N L$, the hyperbolic linear approximation (12) with linear boundary conditions (17) and (19) referred as $H S L$ and the augmented discrete-time linear approximate system (32) which is referred as $A D L S$. For these three models, it is imposed an initial condition and it is verified the steady-state convergence. To this end, it is considered control inputs $U_{1}^{*}=150 \mathrm{rpm}$ and $U_{2}^{*}=$ 300 watts which yield steady-state values $\rho^{*}=1.1 \mathrm{~kg} / \mathrm{m}^{3}$ (or, equivalently, $T^{*}=320.92 \mathrm{~K}$ ), $v^{*}=2.02 \mathrm{~m} / \mathrm{s}$ and $p^{*}=1$ Bar (or, equivalently, $1 \times 10^{5} \mathrm{~Pa}$ in SI units). Figure 3 shows the time response of temperature, speed and pressure at the tube outlet, and the heating column temperature (tube inlet) for all three models. The initial condition was set to $\rho=1.1614 \mathrm{~kg} / \mathrm{m}^{3}, v=1.8 \mathrm{~m} / \mathrm{s}$ and $p=1$ Bar. Notice when converting the value of the initial condition $\rho=1.1614 \mathrm{~kg} / \mathrm{m}^{3}$ in temperature for $H S N L$ using (7) that we have obtained $304 K$ and for the linear approximate models using the linear relationship (20) or (27), we have obtained $301.4 \mathrm{~K}$. It turns out that all models have converged to the expected values.
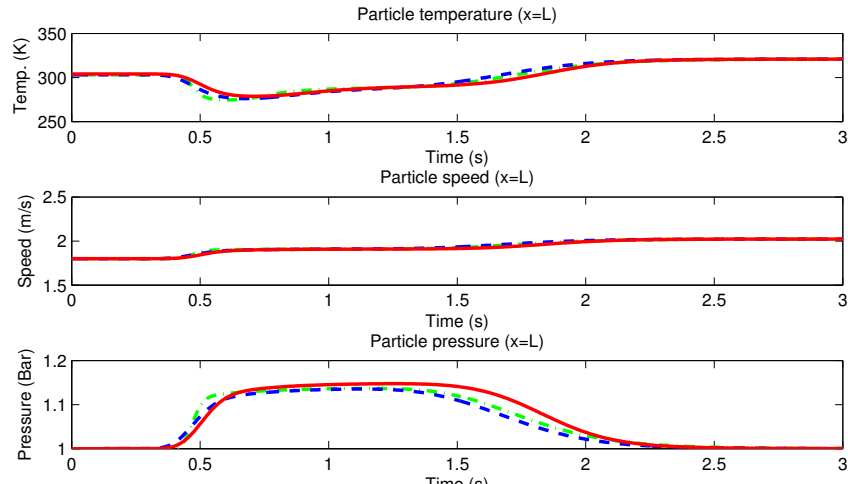

Heating Column Temperature, $(x=0)$

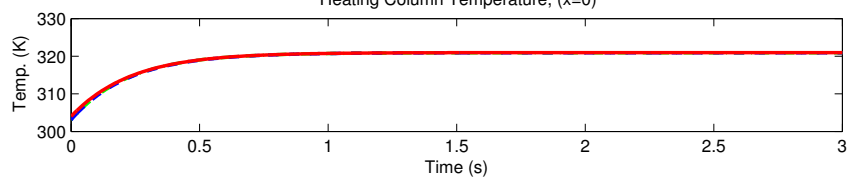

Fig. 3. Temperature, speed and pressure at $x=L$ and temperature at $x=0$ (i.e., heating column temperature) time responses for the models $H S N L(-), H S L(---)$, and $A D L S(---)^{-}$.

Now, a controller for tracking a constant reference temperature at $x=L$ is designed (considering the discretetime linear approximate model $A D L S$ ) by adapting the control law proposed in (34) with (35). To this end, a discrete-time integrator is added to the system dynamics of (32) leading to the following state space realization:

$$
\tilde{\Omega}:\left\{\tilde{\mathcal{Z}}(j+1)=\tilde{A} \tilde{\mathcal{Z}}(j)+\tilde{B} U(j)+B_{r} T_{r e f}\right.
$$

where $\tilde{\mathcal{Z}}(j)=\left[\begin{array}{ll}\mathcal{Z}(j)^{T} & \xi(j)\end{array}\right]^{T}$ is the enlarged state vector, $\xi(j)$ is the integrator state, and

$$
\tilde{A}=\left[\begin{array}{cc}
A & \mathbf{0} \\
-C_{r} & 1
\end{array}\right], \tilde{B}=\left[\begin{array}{c}
B \\
\mathbf{0}
\end{array}\right], B_{r}=\left[\begin{array}{l}
\mathbf{0} \\
1
\end{array}\right],
$$

with $C_{r}$ and $T_{r e f}$ being respectively a constant matrix such that $\bar{T}_{d}(L, j)=C_{r} \mathcal{Z}(j)$ and the desired temperature at $x=L$. Then, the control law (34) is modified to be as follows:

$$
U(j)=\tilde{K} \breve{\mathcal{Z}}(j),
$$

where $\tilde{K}=\left[\begin{array}{ll}K & K_{r}\end{array}\right]$ and $\breve{\mathcal{Z}}(j)=\left[\begin{array}{ll}\hat{\mathcal{Z}}(j)^{T} & \xi(j)\end{array}\right]^{T}$. Figure 4 shows a comparison of the hyperbolic linear PDE model $H S L$, as in (12), with linear boundary conditions (17)-(19) (- - ) and the discrete-time approximate model $A D L S C$ (-.---) using in both cases a controller designed for the $A D L S C$ model. Notice that the controller is able to track the reference temperature $T_{\text {ref }}=325 \mathrm{~K}$ considering both models demonstrating that the designed control law can be applied for boundary control of hyperbolic PDE systems at the cost of some performance degradation.

\section{Concluding Remarks}

In this paper, we have presented the boundary control of first order hyperbolic PDE systems associated to dynamic boundary conditions. Classical techniques such as linear approximation and discretization have been applied to an experimental setup (consisting of a tube, heating column and ventilator) leading to an augmented discrete-time linear approximate model. Then, an output 


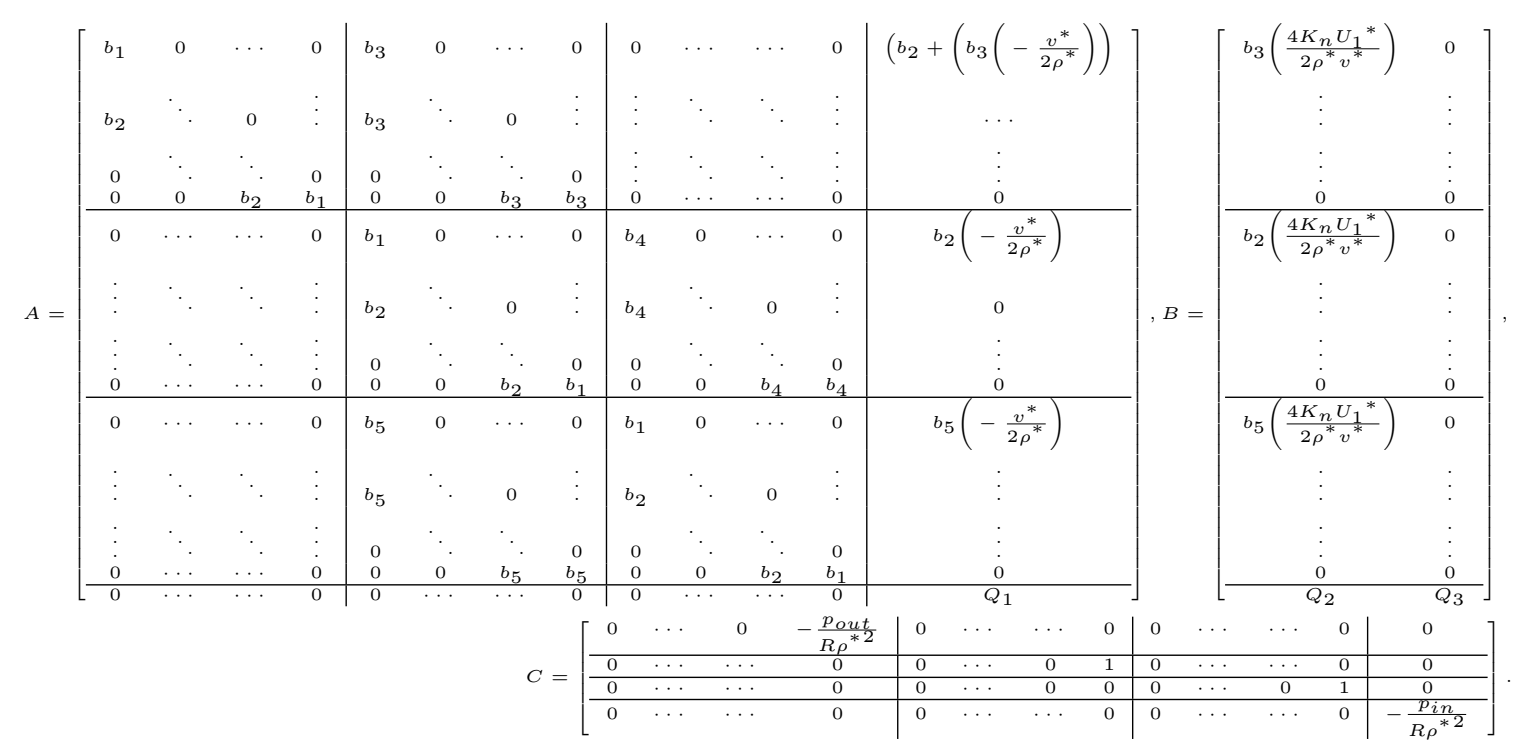

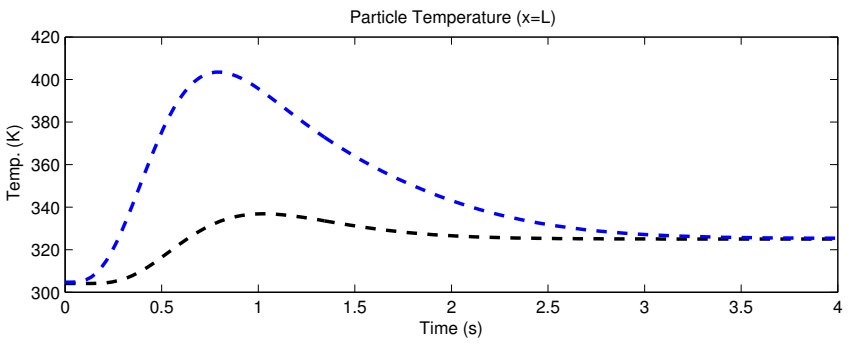

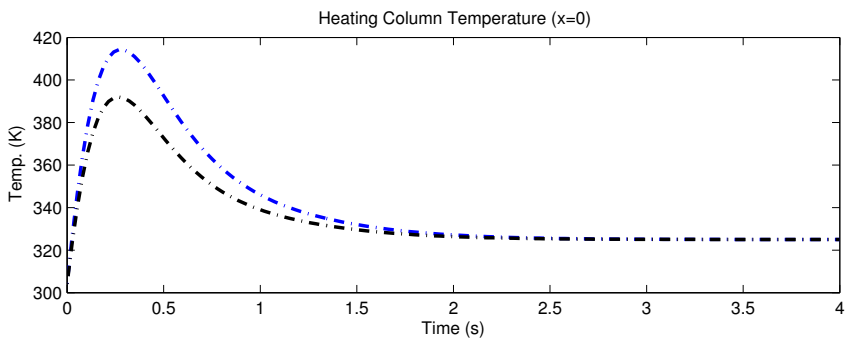

Fig. 4. Time evolution of temperature in $x=L$ and $x=0$ for $H S L$, using the designed control for $A D L S C(---)$ and the $A D L S C$, $(-\cdot-\cdot-)$.

feedback plus integrator control law is derived to track a constant reference temperature. Simulation results have demonstrate the potentials of the proposed approach. Future research will be concentrated in applying these results on the experimental setup.

\section{REFERENCES}

[1] M.K. Banda, M. Herty, and A. Klar. Gas flow in pipeline networks. Networks and Heterogeneous media, 1(1):41-56, 2006.

[2] G. Bastin, J-M. Coron, and B. d'Andréa Novel. On Lyapunov stability analysis of linearized saint-venant equations for a sloping channel. Networks and Heterogeneous Media, 4(2):177-187, 2009.

[3] G. Bastin, B. Haut, J-M. Coron, and B. d'Andréa Novel. Lyapunov stability analysis of networks of scalar conservation laws. Networks and Heterogeneous media, 2(4):749-757, 2007.

[4] F. Castillo, E. Witrant, and L. Dugard. Dynamic boundary stabilization of linear parameter varying hyperbolic systems: Application to a poiseuille flow. IFAC Joint conference SSSC - 11 ${ }^{\text {th }}$ Workshop on Time-Delay Systems, Grenoble: France, 2013.
[5] F. Castillo, E. Witrant, C. Prieur, and L. Dugard. Dynamic boundary stabilization of linear and quasi-linear hyperbolic systems. 51 ${ }^{\text {th }}$ IEEE Control and Decision Conference, 2012.

[6] F. Castillo, E. Witrant, C. Prieur, and L. Dugard. Boundary observers for linear and quasi-linear hyperbolic systems with application to flow control. Automatica, 49(11):3180-3188, 2013.

[7] J-M. Coron, G. Bastin, and B. d'Andréa Novel. A strict Lyapunov function for boundary control of hyperbolic systems of conservation laws. IEEE Transactions on Automatic Control, $52: 2-11,2007$.

[8] J-M. Coron, G. Bastin, and B. d'Andréa Novel. Dissipative boundary conditions for one-dimensional nonlinear hyperbolic systems. SIAM Journal of Control and Optimization, 47(3):1460-1496, 2008.

[9] R. Courant, K. Friedrichs, and H. Lewy. On the partial difference equations of mathematical physics. IBM Journal, pages 215-234, 1967.

[10] J. Halleux. Boundary Control of Quasi-Linear Hyperbolic Initial Boundary-Value Problems. Phd thesis, Universitaires de Louvain, Louvain la Neuve, Belgique, 2004.

[11] F. McQuiston, J. Parker, and J. Spitler. Heating, ventilating \& air conditioning: Analysis and design. Wiley, 2005.

[12] C. Prieur, J. Winkin, and G. Bastin. Robust boundary control of systems of conservation laws. Mathematics of Control, Signals and Systems, 20(2):173-197, 2008.

[13] V. Dos Santos, G. Bastin, J-M. Coron, and B. d'Andréa Novel. Boundary control with integral action for hyperbolic systems of conservation laws. Automatica, 44(1):1310-1318, 2008.

[14] V. Dos Santos and C. Prieur. Boundary control of open channels with numerical and experimental validations. IEEE Transactions on Control Systems Technology, 16(6):12521264, 2008.

[15] E. Witrant, A. D'Innocenzo, G. Sandou, F. Santucci, M.D. Di Benedetto, A.J. Isaksson, K.H. Johansson, S.I. Niculescu, S. Olaru, E. Serra, S. Tennina, and U. Tiberi. Wireless ventilation control for large-scale systems: the minimal industrial case. International Journal of Robust and Nonlinear Control, 20:226-251, january 2010.

[16] E. Witrant, K. Johansson, and HynX team. Air flow modelling in deep wells: aplication to mining ventilation. $4^{\text {th }}$ IEEE Conference on Automation Science and Engineering, august 2008. 